\title{
CONCORDANCIA COPULATIVA, PRONOMBRES SUJETO Y ADQUISICIÓN DE SISTEMAS NO NATIVOS
}

\begin{abstract}
El presente artículo investiga la actuación de distintos pronombres sujeto en la adquisición de un sistema no nativo. Por un lado, estudiamos la evolución en el proceso adquisitivo de los sujetos referenciales. Por otro, analizamos algunas construcciones con sujetos pleonásticos, que incluyen oraciones existenciales y construcciones de concordancia copulativa. Tras realizar varias pruebas a estudiantes españoles de inglés, pudimos determinar cómo el proceso adquisitivo difiere con respecto a los distintos sujetos. Los pronombres pleonásticos fueron los últimos en adquirirse, sobre todo en aquellos contextos que resultan semánticamente complejos para los hablantes nativos de español, como es el caso de las construcciones de concordancia copulativa. Estos resultados harán que debamos replantearnos algunas teorias sobre el valor de los sujetos pleonásticos en la reestructuración del parámetro de Sujeto Nulo.
\end{abstract}

Dentro del marco de la teoría de la Rección y el Ligamiento (Chomsky 1981, 1982), el modelo lingüístico de los Principios y Parámetros establece que toda lengua se adquiere gracias a una Gramática Universal, que todo ser humano posee innatamente. Esta Gramática Universal consta de unos principios o reglas, que son universales, y que ayudan a que la adquisición de toda lengua se lleve a cabo de forma rápida, sin tener que realizar continuas hipótesis sobre la misma. Según esta teoría, algunos de estos principios universales están parametrizados, es decir, poseen un número determinado de valores que deben fijarse a través de evidencia positiva. El ser humano debe seleccionar aquellas opciones que se ajusten a los datos aportados por el entorno. Uno de los parámetros que mayor controversia provocan es el llamado Parámetro Pro-drop o de Sujeto Nulo (Chomsky 1981, Jaeggli \& Safir 1987), que recientemente ha sido reformulado como Parámetro de Argumento Nulo (Hyams 1994). Este parámetro establece una división tipológica entre lenguas, según admitan sujetos fonológicamente nulos o no. ${ }^{1}$ Lenguas como el inglés o el francés no admiten la omisión de sujetos referenciales.

1 Dentro de las propiedades que forman parte del Parámetro Pro-drop, algunos estudiosos han incluido otras características, como son la posibilidad de contar en español con sujetos postverbales,

Pedro vino ayer.

Vino Pedro ayer.

- Peter came yesterday.

*Came Peter yesterday.

o el llamado filtro que-h, que admite extraer un sujeto de una oración subordinada encabezada por el complementante que.

- ¿Quién dices que va a venir?

Who do you say is going to come?

*Who do you say that is going to come? 
(1) He came yesterday.

*Came yesterday.

(2) Il est venu hier.

*Est venu hier.

El español, por otro lado, no requiere la presencia de sujetos referenciales. ${ }^{2}$

(3) Vino ayer.

No puedo ir a tu fiesta.

Además, en inglés o francés encontramos elementos pleonásticos con función de sujeto, mientras que en español éstos no existen.

(4) It rains.

*Rains.

(5) Il pleut.

*Pleut.

(6) Llueve.

*Ello llueve.

El objeto de nuestro artículo es analizar el comportamiento de estas propiedades en la adquisición de un sistema no nativo. Vamos a estudiar el proceso adquisitivo en inglés por parte de hablantes nativos de español, para comprobar posibles implicaciones de unas características con respecto a otras.

Dentro de estas características incluiremos, por un lado, los sujetos referenciales, y por otro los sujetos expletivos, que los dividiremos en distintas categorías: sujetos existenciales,

(7) There is a book on the table.

*Is a book on the table.

(8) Hay un libro en la mesa.

*Ello hay un libro en la mesa.

sujetos expletivos,

(9) It is easy to pass the exam.

*Is easy to pass the exam.

(10) Es fácil aprobar el examen.

*Ello es fácil aprobar el examen.

No obstante, estas características se pueden explicar por reglas de movimiento: Regla $\mathrm{R}$ o affix hopping en el caso de los sujetos postverbales (Chomsky 1981, Hyams 1986) y el Principio de la Categoría Vacía en el caso del efecto que-h (Chomsky 1981), que establece que la variable $h$ debe estar regida y el complementante that del inglés no la puede regir. Por lo tanto, y ya que pueden surgir divergencias al respecto, nos vamos a centrar en aquellas características centrales al parámetro en cuestión.

2

Las oraciones con sujetos referenciales en español únicamente se utilizan por énfasis o para marcar algún cambio en el discurso. Si no cumplen ninguna función pragmática, tienden a ser eliminadas. 
y construcciones de concordancia copulativa, que están formadas por un sujeto expletivo y un objeto en acusativo.

(11) It was him who told me.

*Was him who told me.

(12) Fue él quien me lo dijo.

*Ello fue él quien me lo dijo.

En nuestra investigación hemos contado con un abanico amplio de estudiantes españoles de inglés de la Universidad Pública de Navarra, en España. Todos ellos formaban parte de un programa de autoaprendizaje, que consistía en la asistencia a clases de inglés durante tres horas a la semana y la posibilidad de trabajar autónomamente en un centro de autoacceso. $\mathrm{Al}$ comienzo del curso escolar, estos estudiantes habían realizado una prueba escrita y una entrevista oral con un tutor para conocer su competencia en inglés, y habían sido en consecuencia distribuidos en cinco niveles, de acuerdo con los existentes en distintos organismos oficiales. Para nuestra investigación, les pedimos que rellenaran un cuestionario con su historial lingüístico donde contestaban preguntas relevantes para la investigación, como los años que habían estado aprendiendo el idioma, los exámenes realizados, las estancias en países de habla inglesa y su conocimiento de otros idiomas, aspectos que eran importantes para tener un conocimiento amplio de todos ellos. Además, nos pareció oportuno que realizaran una prueba paralela a la llevada a cabo al inicio del curso, para reafirmar el nivel establecido en cada uno de los casos. Para ello, les pedimos que completaran un test de cloze, ya que este tipo de pruebas son fáciles de administrar y muestran unos resultados globales (Oller 1991, Fotos 1991).

Después de realizar las distintas pruebas, seleccionamos un total de 150 alumnos, 61 mujeres y 89 hombres, y los subdividimos en cinco niveles según su nivel de competencia lingüística.
Nivel 1: 29 alumnos
Nivel 2: 49 alumnos
Nivel 3: 34 alumnos
Nivel 4: 20 alumnos
Nivel 5: 18 alumnos

Los estudiantes debían traducir veintiocho oraciones que ejemplificaban aquellos aspectos que nos interesaba analizar. Además, incluimos oraciones afirmativas, negativas, interrogativas, y subordinadas para contar con un muestreo amplio de todas las propiedades. Con la traducción de estas oraciones podíamos observar los errores que los estudiantes cometían y éstos mostrarán las reglas innatas que se utilizan.

Los resultados en la prueba de traducción fueron los siguientes: 


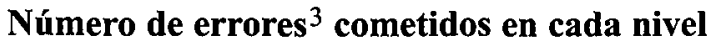

$\begin{array}{lrrrrr} & \text { N1 } & \text { N2 } & \text { N3 } & \text { N4 } & \text { N5 } \\ \text { Suj. referenciales } & 8 & 12 & 5 & 0 & 0 \\ \text { Existencial "there" } & 1 & 18 & 4 & 1 & 0 \\ \text { Expletivo "it". } & 18 & 21 & 18 & 6 & 6 \\ \text { Conc. Copulativa } & 91 & 140 & 83 & 34 & 30\end{array}$

Número medio de errores cometidos en cada nivel

\begin{tabular}{lrrrrr} 
& $\mathrm{N} 1$ & $\mathrm{~N} 2$ & $\mathrm{~N} 3$ & \multicolumn{1}{c}{ N4 } & N5 \\
Suj. referenciales & .28 & .24 & .15 & 0.00 & 0.00 \\
Existencial "there" & .03 & .37 & .12 & .05 & 0.00 \\
Expletivo "it" & .62 & .43 & .53 & .30 & .33 \\
Conc. Copulativa & 3.14 & 2.86 & 2.44 & 1.70 & 1.67
\end{tabular}

\section{Diferencial de errores cometidos en el paso de un nivel a otro}

$\begin{array}{lrrrr} & \mathrm{I} 12 & \mathrm{I} 23 & \mathrm{I} 34 & \mathrm{I} 45 \\ \text { Suj. referenciales } & .03 & -.10 & -.15 & 0.00 \\ \text { Existencial "there" } & .33 & -.25 & -.07 & -.05 \\ \text { Expletivo "it" } & -.19 & .10 & -.23 & .03 \\ \text { Conc. Copulativa } & -.28 & -.42 & -.74 & -.03\end{array}$

Si comparamos el funcionamiento de los sujetos referenciales y los sujetos pleonásticos, vemos cómo ambos siguen un desarrollo desigual. En el primer caso apreciamos un movimiento decreciente a lo largo de los niveles, hasta llegar al nivel 4 de adquisición, donde ya no encontramos ningún error con este tipo de sujetos. Sin embargo, las distintas construcciones con sujetos pleonásticos muestran una evolución diferente. El número de omisiones en su conjunto es significativamente mayor al existente en el caso de los pronombres referenciales. Como afirmábamos anteriormente, los sujetos pleonásticos no existen en español y los sujetos pronominales únicamente se utilizan para cumplir con alguna función pragmática. No obstante, estos sujetos pleonásticos nunca pueden marcar otra función que no sea la puramente gramatical. No pueden mostrar énfasis ni marcar un cambio en el discurso,

(13) She is not coming, he is.

*It's not snowing, it's raining.

(14) It doesn't seem that we are crazy, it seems that you are.

*It doesn't seem that we are crazy, it seems that you are. ${ }^{4}$

3 Al hablar de errores, aludimos a omisiones de los distintos elementos con función de sujeto en contextos obligatorios.

4 Por medio de la letra cursiva, señalamos aquellos elementos que están acentuados. 
Así pues, estos sujetos son eliminados en español..$^{5}$ Cuando los estudiantes españoles adquieren una segunda lengua, en este caso el inglés, transfieren la información existente en su lengua materna, y asumen que estos sujetos pleonásticos no se encuentran en la lengua meta, omitiéndolos de forma más continuada que los sujetos referenciales, que sí existen en la lengua fuente. Los estudiantes irán utilizándolos de forma progresiva, aunque su dominio se verá postergado hasta los estadios más avanzados del proceso adquisitivo.

La adquisición tardía de los pronombres expletivos va a hacer que debamos replantearnos su papel en una segunda lengua. Algunos estudiosos (Hyams 1986, 1989, en la adquisición de una primera lengua y Hilles 1986 en la adquisición de una segunda) afirman que los pronombres expletivos son de vital importancia a la hora de reajustar el valor del parámetro de Sujeto Nulo: los niños ingleses comprenden que existen unos pronombres cuya función es meramente gramatical y éstos provocan el reajuste del parámetro, haciendo que comiencen a utilizar algunas de las características que hasta entonces han omitido, como es el caso de los sujetos referenciales. Sin embargo, los estudiantes españoles de inglés no siguen este mismo planteamiento a la hora de adquirir el inglés como segunda lengua. Los sujetos expletivos no van a determinar la fijación del parámetro sino que se van a adquirir con posterioridad, cuando se hayan controlado el resto de pronombres sujeto.

Además, ambos pronombres pleonásticos no se comportan de igual forma. El número de omisiones en el caso del expletivo it es bastante mayor al existente con el existencial there. A nuestro modo de ver, existen dos causas que explican este comportamiento. Por un lado, los estudiantes españoles conocen el locativo there, que es uno de los primeros en aprenderse en el aula, y también en adquirirse tanto en las primeras como en las segundas lenguas. ${ }^{6}$ Bloom, Lightbown y Hood (1975) muestran ejemplos tempranos del uso del locativo there, que suele ir acompañado de elementos no verbales.

(15) There birdie (el niño señala al mismo tiempo un pájaro)

There is book (señalando un libro)

Baby there (el niño mira dentro de la cuna)

Tanto los niños en el proceso adquisitivo de su lengua materna como los adultos que adquieren un segundo idioma dominan el uso del locativo there con celeridad. Este conocimiento hace que el existencial there les resulte más familiar, e incluso es posible que confundan el locativo y el existencial en los niveles inferiores de adquisición, intercambiándolos. Utilizan oraciones como

(16) There is a beautiful girl.

5 El Principio de Elisión del Pronombre establece que todo elemento pronominal léxico debe eliminarse si un elemento pronominal nulo es posible (Chomsky 1981).

6 Cuando hablamos de una segunda lengua, nos referimos a toda aquélla que se adquiere con posterioridad a la lengua materna. 
y pueden significar que "hay una niña guapa" o que "allí hay una niña guapa", enfatizando el lugar en el que se encuentra.

Por otro lado, la enseñanza del existencial desarrollada en el aula puede igualmente haber influido en su adquisición. En los programas educativos españoles, el existencial siempre viene acompañado del verbo copulativo be (there is/there are). Los estudiantes aprenden ambas partículas de forma conjunta, e inconscientemente las vinculan dentro de un mismo constituyente. De este modo, les resulta difícil eliminar un elemento de los dos que configuran un todo. Este hecho puede también haber influido en el reducido número de omisiones que encontramos con el existencial en el primer nivel de adquisición. Los estudiantes han conocido el sujeto expletivo there en ese mismo período, y lo usan con más asiduidad por estar reciente su aprendizaje.

Sin embargo, no debemos magnificar la influencia de la enseñanza en el aula. Si ésta fuera tan importante, no podríamos explicar el elevado número de errores existentes en el caso de los pronombres expletivos con respecto a los pronombres referenciales. Los primeros se utilizan frecuentemente ya en los primeros niveles adquisitivos y nunca se pueden omitir, mientras que los sujetos referenciales pueden eliminarse en situaciones especiales, como en contextos informales o en cartas y diarios. ${ }^{7} \mathrm{Si}$ la función de la enseñanza en el aula fuera tan importante, no entenderíamos por qué el número de errores es mayor en el caso de los pronombres expletivos, que aparecen de forma obligatoria y temprana en la enseñanza de idiomas.

De entre todas las características analizadas, el mayor número de errores lo encontramos en el caso de las construcciones de concordancia copulativa. Estas construcciones resultan complejas para los estudiantes, al constar de un elemento expletivo con función de sujeto, que como hemos visto anteriormente se adquieren de forma tardía, y de un objeto en acusativo. Su adquisición se verá postergada, y existe un porcentaje significativamente alto de omisiones incluso en los niveles superiores del proceso adquisitivo.

\section{Conclusiones}

En esta investigación hemos analizado el comportamiento de algunos pronombres sujeto en la adquisición del inglés como segunda lengua. Nuestros resultados indican que aquellas construcciones con sujetos pleonásticos son las últimas en adquirirse. De entre ellas, las construcciones de concordancia copulativa son las que más problemas presentan, al resultar semánticamente complejas para los estudiantes. Además, encontramos diferencias significativas en el uso de los sujetos existenciales y expletivos, que pueden parcialmente explicarse por el planteamiento didáctico desarrollado en el aula.

7 Rizzi (1994) y Haegeman $(1989,1990)$ señalan que el elemento que se omite en construcciones informales o en diarios no es pro. Se trata de una constante nula que no es pronominal, sino una opción vacía que se identifica por el contexto. 
La adquisición tardía de los sujetos pleonásticos hará que debamos revisar algunas teorías sobre el valor de los sujetos pleonásticos en la reestructuración del parámetro del Sujeto Nulo.

\section{Bibliografia}

Bloom, L., Lightbown, P. \& L. Hood. (1975). Structure and varitation in child language, Monograph of the Society for Research in Child Development, 40, 2.

CHOMSKY, N. (1982). Some concepts and consequences of the theory of Government and Binding. Linguistic Inquiry Monograph, MIT Press, Cambridge: Mass.

CHOMSкy, N. (1981). Lectures on government and binding. Dordrecht: Foris.

Foros, S. (1991). "The cloze test as an integrative measure of EFL proficiency: A substitute for essays on college entrance examinations?". Language Learning 41, 313-336.

HAEGEMAN, L. (1989). Understood subjects in English diaries. On the relevance of theoretical syntax for the study of register variation, ms. Universidad de Ginebra.

HaEgeman, L. (1990). "Non-overt subjects in diary contexts". En J. Mascaro \& N. Nespor (eds), Grammar in progress: GLOW Essays for Henk van Riemsdijk (167-174). Dordrecht: Foris.

Hilles, J. (1986). "Interlanguage and the pro-drop parameter". Second Language Research 2, 33-52.

HYAMS, N. (1986). Language acquisition and the theory of parameters. Dordrecht: Reidel.

HYams, N. (1989). "The Null subject parameter in language acquisition". En O. Jaeggli \& K. Safir (eds), The null subject parameter (215-238). Dordrecht: Reidel.

HYAMS, N. (1994). "V2 null arguments and COMP projections". En Hoekstra, T. \& B. Schwartz), Language acquisition studies in generative grammar. Amsterdam: John Benjamins.

JAEGGLI, O. \& K. SAFIR. (1987). The null subject parameter. Dordrecht: Reidel.

OLLER, J., Jr. (1991). "Foreign language testing: I: Its breadth; II: Its depth". ADFL Bulletin 22; 23, 33-38/5-13.

RızzI, L. (1994). "Early null subjects and root null subjects". En Hoekstra, T. \& B. Schwartz (eds), Language acquisition studies in generative grammar. Amsterdam: John Benjamins.

RUIZ DE ZAROBE, Y. (1995). La actuación del parámetro pro-drop en la adquisición del inglés como segunda lengua, tesis doctoral. Universidad del Pais Vasco, .

RUIZ DE ZAROBE, Y. (1996). “Expletive pronouns and the readjustment of the pro-drop parameter". The Forum of Phi Sigma lot, International Foreign Language Honor Society, University of Nevada, 18(1), 10-11.

RUIZ DE ZAROBE, Y. (1998). "El parámetro pro-drop y la adquisición de segundas lenguas". ITL: Review of Applied Linguistics, 121-122.

RUIZ DE ZAROBE, Y. (1998). "Uniformidad morfológica y adquisición de sujetos en inglés lengua extranjera". Langues et Linguistique, 24. 


\section{SKLADNOST V VEZAVI, ZAIMKI KOT OSEBEK IN USVAJANJE TUJIH JEZIKOVNIH SISTEMOV}

Prispevek skuša osvetliti pojavljanje zaimka kot osebek ob usvajanju sistema v tujem jeziku. $\mathrm{Z}$ ene strani se tehta raba referenčnih osebkov, $\mathrm{z}$ druge pa se analizira strukture, kjer se pojavlja ob glagolu osebni zaimek kot slovnični osebek. Primerja se sistem s španščini s tistim v angleščini, deloma tudi v francoščini: španščina ne dovoljuje osebnega zaimka, npr., v izjavi llueve (*ello llueve), taka zgradba pa je v angleščini obvezna, it rains, tudi francosko il pleut. Avtorica ugotavlja tri različne zgradbe, ki so $v$ španščini drugačne kot $v$ angleščini; njena anketa je bila izvedena na gradivu, ki ga je nudilo učenje angleščine, in s tem težave, ki so jih imeli španski študenti. Pri tem je prišla do spoznanja, da so bile ravno strukture s slovničnim osebkom $v$ angleščini tiste, ki so jih španski govorci usvojili kot zadnje, zlasti takrat, ko je bil stavek pomensko močno zapleten. S tega vidika bo koristno na novo pretehtati teorijo o slovničnih osebkih, oziroma o ničtem osebku. 